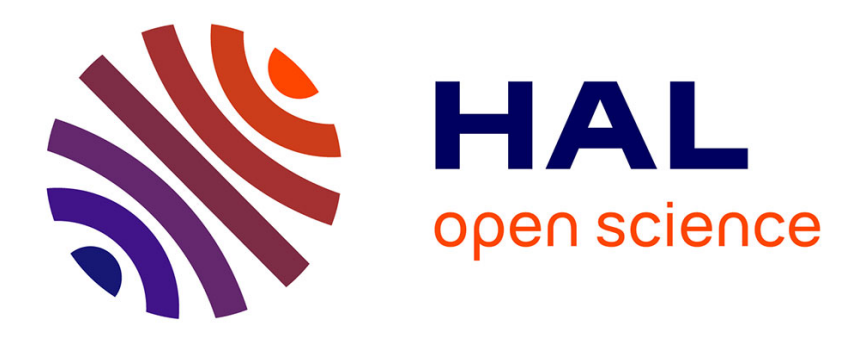

\title{
Micromorphic modelling of grain size effects in metal polycrystals
}

Nicolas Cordero, Samuel Forest, Esteban P. Busso

\section{To cite this version:}

Nicolas Cordero, Samuel Forest, Esteban P. Busso. Micromorphic modelling of grain size effects in metal polycrystals. GAMM-Mitteilungen, 2013, 36 (2), pp.186-202. 10.1002/gamm.201310011. hal-00904368

\section{HAL Id: hal-00904368 \\ https://hal-mines-paristech.archives-ouvertes.fr/hal-00904368}

Submitted on 11 Jul 2018

HAL is a multi-disciplinary open access archive for the deposit and dissemination of scientific research documents, whether they are published or not. The documents may come from teaching and research institutions in France or abroad, or from public or private research centers.
L'archive ouverte pluridisciplinaire HAL, est destinée au dépôt et à la diffusion de documents scientifiques de niveau recherche, publiés ou non, émanant des établissements d'enseignement et de recherche français ou étrangers, des laboratoires publics ou privés. 


\title{
Micromorphic modelling of grain size effects in metal polycrystals
}

\author{
N. M. Cordero ${ }^{1}$, S. Forest $^{1 *}$, and E. P. Busso ${ }^{1}$ \\ ${ }^{1}$ Centre des Matériaux, Mines ParisTech, CNRS UMR 7633, BP 87, 91003 Evry, France
}

Received 13 October 2012, revised 24 January 2013, accepted 19 February 2013

Published online 07 October 2013

Key words Crystal plasticity, strain gradient, micromorphic theory, grain size effect, polycrystal,homogenization

MSC (2010) 74-xx,74A-xx,74Q-xx

A long-standing problem in the modelling of polycrystal behavior from the knowledge of the constitutive equations of single crystals is the incorporation of grain size effects. Standard homogenization procedures efficiently take into account grain to grain interaction primarily induced by strain incompatibilities at grain boundary and grain morphology effects. Continuum crystal plasticity models are available that satisfactorily describe dislocation forest hardening associated with multiplication and dynamic recovery of dislocation populations. One strong limitation of these techniques is that the local crystal plasticity constitutive parameters at the grain scale are assumed to be known. Conversely, these material parameters can be identified from the macroscopic polycrystal response by an inverse approach involving the homogenization model. But then the found crystal plasticity model will be valid only for the considered grain size and the model can hardly be used for prediction of polycrystal behavior for another grain state of the material. Generalized continuum approaches arise as the suitable mechanical framework for formulating enhanced crystal plasticity models that intrinsically include grain size dependent local material responses. They incorporate the dislocation density tensor as a consitutive variable. Recent developments have shown the limitations of existing strain gradient models in the case of a two-phase laminate microstructure under single or double slip [1]. They plead for a theory even more general than the strain gradient or Cosserat approach, namely the micromorphic model presented here. The effect of the dislocation density tensor is introduced into the classical crystal plasticity framework by means of a micromorphic theory of single crystals. A computational homogenization strategy is presented in order to simulate the global and local responses of two-dimensional polycrystalline aggregates for grain sizes ranging from 1 to 200 microns. The model is shown to induce a size-dependent kinematic hardening component which is responsible for the observed strong size effects. The yield stress at a given averaged plastic deformation is shown to follow a power law scaling relation for grain sizes larger than a critical grain size. The field of plastic deformation is also strongly affected by grain size, micron-size grains leading to the formation of intense slip bands crossing several grains.

(c) 2013 WILEY-VCH Verlag GmbH \& Co. KGaA, Weinheim

* Corresponding author E-mail: samuel.forest@ensmp.fr, Phone: +33 160763 051, Fax: +33 160763150 


\section{Introduction}

The prediction of plastic strain fields inside polycrystals based on classical continuum crystal plasticity as considered in the finite element simulations of polycrystalline aggregates is insensitive to absolute grain size [2-5]. In such full field simulations, it is thought that the absolute grain size should not be introduced explicitly into the constitutive equations since, in a field theory, the material point is not aware of the size of the grain it is sitting in. Rather, the grain size effect should arise from information about gradient fields, for instance through the dislocation density tensor which is directly related to the plastic strain gradient. Finite element simulations of polycrystalline aggregates based on strain gradient plasticity models have revealed significant grain size effects, at least in a qualitative manner [6-8]. However, evidence that strain gradient plasticity models are able to predict the Hall-Petch behaviour from full field simulations remains seldom.

The strain gradient effects do not affect the overall behaviour only but also the intragranular fields of plastic deformation and stresses. Little evidence of this fact has been provided in the literature from the computational side except recently in $[9,10]$ where it was shown that strain localization phenomena can occur in small grains in the form of intense slip bands associated with little contributions of the dislocation density tensor. Experimental evidence of such phenomena remains rather difficult to provide since large grain size ranges for the same material and texture are necessary, which is rarely the case in practice.

The purpose of this work is to present a generalised continuum model, the microcurl model, and to apply it to simulate the behaviour of polycrystalline aggregates with various microstructure sizes. It will be shown that a significant size dependent linear kinematic hardening coming from the dislocation density tensor arises and that simple constitutive equations lead to Hall-Petch like polycrystal behaviour provided that some intrinsic material parameters are identified. This model shares several common features with available strain gradient plasticity models. It is a micromorphic crystal plasticity approach which belongs to the class of generalised continuum models with additional degrees of freedom as presented in $[11,12]$. It was proposed by Cordero et al. [1] to regularise the response in the presence of material interfaces of the strain gradient plasticity theory from [13] which includes the full curl of the plastic deformation tensor $\boldsymbol{H}^{p}$.

The microcurl model is presented for crystal plasticity in section 2, within the framework of small deformation theory. The reader is referred to the work [14] for the development of the theory at finite deformation. The continuity requirements at grain boundaries are introduced in section 3 and their role in the size effects is studied. As an illustration, finite element simulations of the simple shear response of a polycrystalline aggregate with a relatively small number of grains ( 24 grains) are first performed to have an overview of the size effects produced by the model. Various microstructure sizes are investigated and a virtual material is considered with various intrinsic length scales. It is shown in particular that, even when no classical isotropic hardening is introduced, the linear kinematic hardening produced by the microcurl model is sufficient to reproduce scaling laws observed in grain size effects. This first set of simulations also serves as a basis for the identification of the intrinsic material parameters. Finally, finite element simulations of the simple shear response of a larger idealised aluminium aggregate are performed in section 4 . The impact of the microstructure size on the overall response is investigated and the question of the dependence of plastic strain fields with respect to grain size is addressed. 
The following notations are used: vectors and second rank tensors are respectively denoted by $\underline{\boldsymbol{a}}$ and $\boldsymbol{a}$; the curl operator in a Cartesian frame for a tensor $\underset{\sim}{\boldsymbol{a}}$ of rank two is

$$
(\operatorname{curl} \underset{\sim}{\boldsymbol{a}})_{i j}=\epsilon_{j k l} a_{i k, l}
$$

where $\epsilon_{i j k}$ is the permutation tensor.

\section{The microcurl model for crystal plasticity}

In the crystal plasticity theory at small deformation, the gradient of the velocity field, $\underline{\dot{u}}$, can be decomposed into the elastic and plastic deformation rates:

$$
\dot{\sim}=\underline{\dot{\boldsymbol{u}}} \otimes \nabla=\dot{\sim}^{e}+\dot{\sim}^{p}
$$

where the plastic deformation rate is the result of slip processes

$$
\dot{\sim}^{p}=\sum_{\alpha}^{n} \dot{\gamma}^{\alpha} \underline{\boldsymbol{l}}^{\alpha} \otimes \underline{\boldsymbol{n}}^{\alpha},
$$

with $n$ the number of slip systems, $\dot{\gamma}^{\alpha}$ the slip rate for the slip system $\alpha, \underline{\boldsymbol{l}}^{\alpha}$ the slip direction and $\underline{\boldsymbol{n}}^{\alpha}$ the normal to the slip plane, $\underline{\boldsymbol{l}}^{\alpha} \otimes \underline{\boldsymbol{n}}^{\alpha}$ being the orientation tensor for the slip system $\alpha$. The elastic deformation $\boldsymbol{H}^{e}$ accommodates the incompatibilities induced by the plastic deformation field $\boldsymbol{H}^{p}$. The curl operator applied to a compatible field gives zero, so we have:

$$
\operatorname{curl} \underset{\sim}{\dot{\boldsymbol{H}}}=\operatorname{curl}{\underset{\sim}{\dot{\boldsymbol{H}}}}^{e}+\operatorname{curl}{\underset{\sim}{\dot{\boldsymbol{H}}}}^{p}=0 .
$$

The dislocation density tensor $[15,16]$ characterises the incompatibility of plastic deformation, it is defined as:

$$
\underset{\sim}{\boldsymbol{\Gamma}}=-\operatorname{curl}{\underset{\sim}{\boldsymbol{H}}}^{p}=\operatorname{curl}{\underset{\sim}{\boldsymbol{H}}}^{e}
$$

The microcurl model originates from the need to include the dislocation tensor into the continuum crystal plasticity framework. It will be used to simulate the response of polycrystalline aggregates with various microstructure sizes. It has been shown by Cordero et al. [1] that this model produces a size-dependent linear kinematic hardening coming from a back-stress contribution of the dislocation density tensor, or equivalently from geometrically necessary dislocations (GNDs). The main characteristics of the generated size effect, namely the maximum extra-hardening, the characteristic size for which size effects occur and the scaling law exponent, can be controlled through two generalised moduli introduced as material parameters.

Note that many authors do not introduce the full dislocation tensor itself in the constitutive modelling but rather the individual contributions of all slip systems in the form of scalar densities of geometrically necessary dislocations, see for instance [13]. The amount of information about all these densities is larger than that contained in $\underset{\sim}{\Gamma}$ but also leads to a higher computational cost and to difficulties regarding interface conditions at grain boundaries, as discussed in [17]. 


\subsection{Balance equations}

The degrees of freedom of the theory are the displacement vector, $\underline{\boldsymbol{u}}$, and the additional plastic micro-deformation variable, $\chi^{p}$, introduced as a generally non-symmetric second-rank tensor.

$$
D O F=\left\{\underline{\boldsymbol{u}}, \underset{\sim}{\chi^{p}}\right\}
$$

The components of $\chi^{p}$ are introduced as independent degrees of freedom, in three-dimension (3D) they are nine, four of them remain in two-dimension (2D). These components are distinct from the components of the plastic deformation, $\boldsymbol{\sim}^{p}$, which are treated as internal variables. The field $\chi^{p}$ is generally incompatible, i.e., it may not derive from a vector field. A first gradient theory is adopted with respect to the degrees of freedom, however we assume that only the curl part of the gradient of the plastic micro-deformation has an effect in the power of internal forces. This is motivated by the definition (5) of the dislocation density tensor. The microcurl continuum is characterised by an enhanced power density of internal forces of the form:

$$
p^{(i)}=\underset{\sim}{\boldsymbol{\sigma}}: \underset{\sim}{\dot{\boldsymbol{H}}}+\underset{\sim}{\boldsymbol{s}}:{\underset{\sim}{\dot{\boldsymbol{\chi}}}}^{p}+\underset{\sim}{\boldsymbol{M}}: \operatorname{curl} \underset{\sim}{\dot{\boldsymbol{\chi}}^{p}}
$$

The stress tensor, $\underset{\sim}{\boldsymbol{\sigma}}$, is symmetric while the micro-stress tensor, $\underset{\sim}{\boldsymbol{s}}$, and the double-stress tensor, $\underset{\sim}{M}$, are generally asymmetric. Then, the total power of internal forces in a domain $V$ with boundary $\partial V$ is:

$$
\begin{aligned}
-\mathcal{P}^{(i)} & =\int_{V} p^{(i)} d V=\int_{V}\left(\underset{\sim}{\boldsymbol{\sigma}}: \underset{\sim}{\dot{\boldsymbol{H}}}+\underset{\sim}{s}:{\underset{\sim}{\dot{\chi}}}^{p}+\underset{\sim}{\boldsymbol{M}}: \operatorname{curl} \underset{\sim}{\dot{\boldsymbol{\chi}}^{p}}\right) d V \\
& =\int_{V}\left(\left(\sigma_{i j} \dot{u}_{i}\right)_{, j}+\left(M_{i j} \epsilon_{j k l} \dot{\chi}_{i k}^{p}\right)_{, l}\right) d V \\
& +\int_{V}\left(-\sigma_{i j, j} \dot{u}_{i}+s_{i j} \dot{\chi}_{i j}^{p}-\epsilon_{j k l} M_{i j, l} \dot{\chi}_{i k}^{p}\right) d V \\
& =-\int_{V} \sigma_{i j, j} \dot{u}_{i} d V-\int_{V}\left(\epsilon_{k j l} M_{i k, l}-s_{i j}\right) \dot{\chi}_{i j}^{p} d V \\
& +\int_{\partial V} \sigma_{i j} n_{j} \dot{u}_{i} d S+\int_{\partial V} \epsilon_{j k l} M_{i j} n_{l} \dot{\chi}_{i k}^{p} d S .
\end{aligned}
$$

The generalised balance of momentum equations are derived from the method of virtual power. In the absence of body forces considered here for the sake of conciseness, we end up with

$$
\operatorname{div} \underset{\sim}{\boldsymbol{\sigma}}=0, \quad \operatorname{curl} \underset{\sim}{\boldsymbol{M}}+\underset{\sim}{\boldsymbol{s}}=0
$$

The corresponding boundary conditions are,

$$
\underline{\boldsymbol{t}}=\underset{\sim}{\boldsymbol{\sigma}} \cdot \underline{\boldsymbol{n}}, \quad \underset{\sim}{\boldsymbol{m}}=\underset{\sim}{M} \cdot \underline{\boldsymbol{\epsilon}} \cdot \underline{\boldsymbol{n}},
$$

where $\underline{t}$ and $\underset{\sim}{\boldsymbol{m}}$ are the simple and double tractions at the boundary, $\underline{\boldsymbol{n}}$ is the unit normal vector to the boundary. The meaning of the plastic micro-deformation, $\bar{\chi}^{p}$, will appear when stating the following constitutive equations. 


\subsection{Constitutive equations}

We introduce the relative plastic strain,

$$
{\underset{\sim}{e}}^{p}:={\underset{\sim}{\boldsymbol{H}}}^{p}-{\underset{\sim}{\chi}}^{p}
$$

measuring the difference between the plastic deformation and the plastic micro-deformation, thus introducing a coupling between plastic micro and macro-deformation. Let us then consider the tensor,

$$
\underset{\sim}{\Gamma}:=\operatorname{curl} \underset{\sim}{\chi^{p}}
$$

which is the curl part of $\chi^{p}$ in the same way as the dislocation density tensor given in Eq. (5) is the curl part of $\boldsymbol{H}^{p}$. The free energy function is assumed to have the following arguments:

$$
\psi\left(\underset{\sim}{\varepsilon^{e}}, \quad \underset{\sim}{e^{p}}, \quad \underset{\sim}{\Gamma}\right) .
$$

where ${\underset{\sim}{\varepsilon}}^{e}$ is the elastic strain, i.e. the symmetric part of ${\underset{\sim}{\boldsymbol{H}}}^{e}$. The reduced entropy inequality reads:

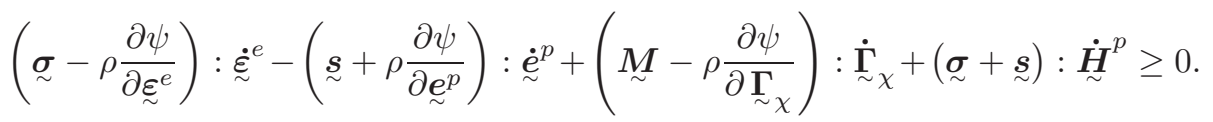

And the following state laws are adopted:

$$
\underset{\sim}{\boldsymbol{\sigma}}=\rho \frac{\partial \psi}{\partial{\underset{\sim}{\boldsymbol{\varepsilon}}}^{e}}, \quad \underset{\sim}{\boldsymbol{s}}=-\rho \frac{\partial \psi}{\partial{\underset{\sim}{\boldsymbol{e}}}^{p}}, \quad \underset{\sim}{\boldsymbol{M}}=\rho \frac{\partial \psi}{\partial \underset{\sim}{\boldsymbol{\Gamma}}},
$$

so that the residual intrinsic dissipation rate remains as

$$
D=(\underset{\sim}{\boldsymbol{\sigma}}+\underset{\sim}{\boldsymbol{s}}): \dot{\sim}^{p} \geq 0
$$

If we consider a viscoplastic potential expressed in terms of the effective stress, $\Omega(\underset{\sim}{\boldsymbol{\sigma}}+\underset{\sim}{\boldsymbol{s}})$, the flow rule reads:

$$
\dot{\sim}_{\sim}^{p}=\frac{\partial \Omega}{\partial(\underset{\sim}{\boldsymbol{\sigma}}+\underset{\sim}{\boldsymbol{s}})}
$$

Assuming a quadratic potential in Eq. (14), the following linear relationships are obtained:

$$
\underset{\sim}{\boldsymbol{\sigma}}=\underset{\approx}{\boldsymbol{\Lambda}}:{\underset{\sim}{\varepsilon}}^{e}, \quad \underset{\sim}{s}=-H_{\chi}{\underset{\sim}{e}}^{p}, \quad \underset{\sim}{\boldsymbol{M}}=A \underset{\sim}{\boldsymbol{\Gamma}},
$$

where $\underset{\approx}{\Lambda}$ is the four-rank tensor of the elastic moduli assumed isotropic in this work, $H_{\chi}$ and $A$ are the generalised moduli. It has already be noted that no dissipative part has been introduced for $\underset{\sim}{\underset{\chi}{\Gamma}}$. The reason is that this simple version of the model is sufficient to highlight relevant size effects, at least in a qualitative manner. Realistic quantitative predictions would require more complex constitutive equations for the generalised stress $\underset{\sim}{M}$ [17-19]. 
For the sake of clarity, let us consider a crystal deforming under single slip conditions. The plastic deformation rate is

$$
\dot{\sim}^{p}=\dot{\gamma} \underline{\boldsymbol{l}} \otimes \underline{\boldsymbol{n}}
$$

and the dissipation takes the form:

$$
D=(\tau+\underset{\sim}{\boldsymbol{s}}:(\underline{\boldsymbol{l}} \otimes \underline{\boldsymbol{n}})) \dot{\gamma} \geq 0 \text {. }
$$

where $\tau=\underset{\sim}{\boldsymbol{\sigma}}: \underline{\boldsymbol{l}} \otimes \underline{\boldsymbol{n}}$ is the resolved shear stress. A generalised Schmid criterion is proposed

$$
|\tau+\underset{\sim}{\boldsymbol{s}}:(\underline{\boldsymbol{l}} \otimes \underline{\boldsymbol{n}})|=\tau_{c},
$$

where $\tau_{c}$ is the critical resolved shear stress taken to be constant (meaning that no strainhardening is considered here for simplicity). A back-stress component naturally arises in the yield function $f(\tau, \underset{\sim}{\boldsymbol{s}})=|\tau+\underset{\sim}{\boldsymbol{s}}:(\underline{\boldsymbol{l}} \otimes \underline{\boldsymbol{n}})|-\tau_{c}$. We can obtain the expression of this back-stress component, $x$, using Eqs. (8) and (17), we have:

$$
x=-\underline{s}:(\underline{\boldsymbol{l}} \otimes \underline{\boldsymbol{n}})=A\left(\operatorname{curl} \operatorname{curl}{\underset{\sim}{\chi}}^{p}\right):(\underline{\boldsymbol{l}} \otimes \underline{\boldsymbol{n}}) .
$$

\subsection{Internal constraint}

An internal constraint controlling the plastic micro-deformation is introduced. The modulus $H_{\chi}$ in Eq. (17) sets a coupling between the macro and micro-variables, it can be seen as a penalty factor constraining the relative plastic deformation, $e^{p}$, to remain sufficiently small. Then, high values of the coupling modulus, $H_{\chi}$, enforce the plastic micro-deformation to be as close as possible to the macroscopic plastic deformation, ${\underset{\sim}{\boldsymbol{H}}}^{p}$. Due to Eq. (11), this internal constraint also links $\underset{\sim}{\Gamma}$ to the dislocation density tensor $\underset{\sim}{\Gamma}$. In what follows, as high values of $H_{\chi}$ are used, ${\underset{\sim}{\sim} \chi}_{\chi}$ is computed and referred as the dislocation density tensor. Moreover it has been shown in [1] that $H_{\chi}$ has a major effect on the scaling law exponent and on the maximum extra-stress obtained analytically on a two-phase periodic microstructure under simple shear. It will be shown later in this work that this effect remains in the case of polycrystalline aggregates. In the limit, an infinite coupling modulus leads to $\chi^{p} \equiv \boldsymbol{\sim}^{p}$ and then the microcurl model is equivalent to more conventional strain gradient plasticity according to [13].

\section{Size effects induced by grain boundaries}

The model is now applied to simulate the response of polycrystals and the effects of the size of their microstructure.

The interface conditions at grain boundaries play a major role in the simulated size effects in the polycrystal behaviour. No special interface law is considered in this work, although such physically motivated interface conditions exist in the literature, see [20]. Instead we consider the canonical interface conditions that arise from the formulation of the balance equations of the microcurl continuum model. These conditions are the continuity of displacement, $\underline{\boldsymbol{u}}$, and the continuity of plastic micro-deformation, $\chi^{p}$. These conditions also include the continuity of the simple and double tractions, $\underline{t}$ and $\underset{\sim}{M}$, described in Eq. (9). Continuity of displacement 
excludes grain boundary cracking and sliding. Continuity of plastic micro-deformation is reminiscent of the fact that dislocations generally do not cross grain boundaries, especially for such random grain boundaries. Note that in the microcurl model, only the kinematic degrees of freedom $\chi^{p}$ are continuous. This is not the case of the plastic deformation, $\boldsymbol{H}^{p}$, which is treated here as an internal variable. However, due to the internal constraint discussed in section 2.3, $\boldsymbol{\sim}^{p}$ closely follows the plastic micro-deformation, so that it is quasi-continuous at grain boundaries when the penalty coefficient, $H_{\chi}$, is high enough. Conversely, lower values of $H_{\chi}$ may allow slightly discontinuous plastic deformation, which may be tentatively interpreted as dislocation sinking inside grain boundaries. The continuity of the associated tractions expresses the transmission of classical and generalised internal forces from one grain to another through grain boundaries. Such continuum models are then able to mimic in that way the development of dislocation pile-ups at grain boundaries [21].

These continuity requirements at grain boundaries represent intermediate conditions between the so-called micro-hard and micro-free conditions used in the literature $[8,22]$. According to the latter, the generalised tractions are set to zero at grain boundary and plastic slip (or dislocation densities) are set to zero at interfaces according to the former conditions.

\subsection{Boundary value problem for polycrystals}

The size effects exhibited by the solution of the boundary value problem are linked to an intrinsic length scale, $l_{s}$, introduced through the generalised moduli $H_{\chi}$ and $A$ of Eq. (17) and defined as:

$$
l_{s}=\sqrt{\frac{A}{H_{\chi}}}
$$

This intrinsic length scale has to be consistent with the fact that plasticity effects occur at scales ranging from hundreds of nanometers to a few microns. In addition, as stated in section 2.3, the coupling modulus, $H_{\chi}$, has to be chosen high enough to ensure that $\underset{\sim}{\boldsymbol{\chi}}$ and $\boldsymbol{H}^{p}$ are close. $H_{\chi}$ also determines the scaling law exponent. These requirements are guidelines for the choice of relevant generalised moduli $H_{\chi}$ and $A$. The sets of material parameters used in this paper are chosen in that way.

The finite element simulations have been made on periodic 2D meshes of periodic polycrystalline aggregates generated by a method based on Voronoi tessellations (Fig. 1(a)(b)). The integration order of elements is quadratic. The Voronoi polyhedra represent the grains, the random distribution of their centers has been controlled so that their sizes are essentially the same, that is why we can reasonably assume that the mean grain size, $d$, is sufficient to characterise the microstructure of our aggregates. A random orientation is assigned to each grain and two slip systems are taken into account. In 2D, the plastic behaviour of f.c.c. crystals can be simulated with 2D planar double slip by considering two effective slip systems separated by an angle of $2 \phi$ [23,24]. Figure 1(c) describes the geometry. The slip system pair is oriented by the angle $\theta$ which is the grain orientation randomly fixed for each grain. For a f.c.c. crystal $\phi=35.1^{\circ}$, it corresponds to the orientation of the close-packed planes in the crystal lattice of the grain. 


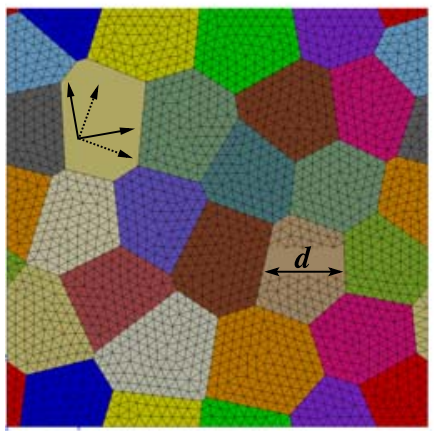

(a)

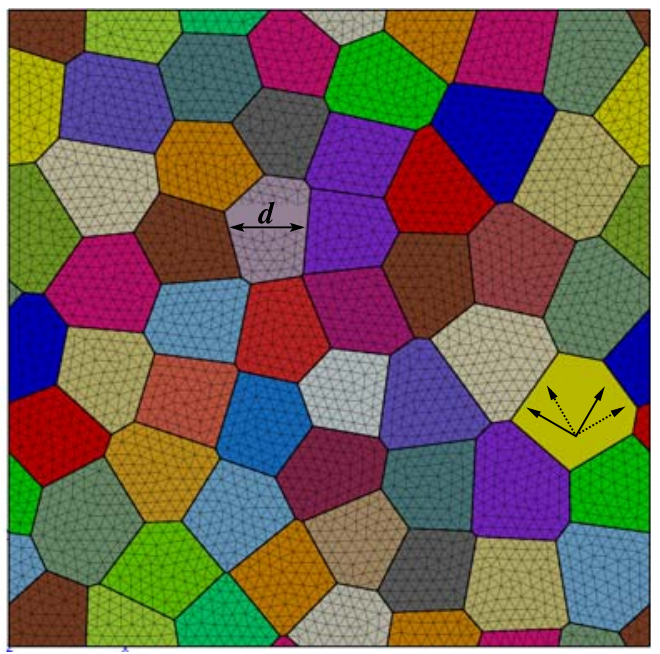

(b)

(c)

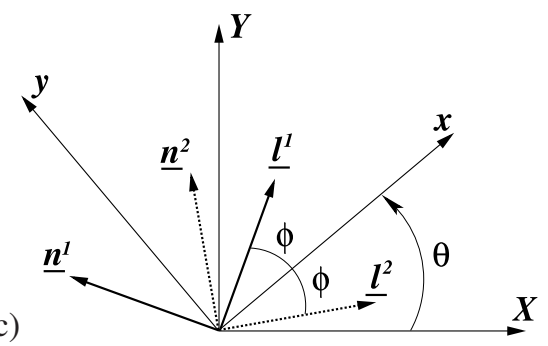

Fig. 1 (online colour at: www.gamm-mitteilungen.org) Periodic meshes of the 2D periodic aggregates used in the finite element simulations: (a) 24 grains, (b) 52 gains. Two slip systems are taken into account in each randomly oriented grain. Various mean grain sizes, $d$, ranging from tens of nanometers to hundreds of microns, are investigated. (c) Description of the two effective slip systems for 2D planar double slip.

Periodic homogenization for generalised continua is used to predict the effective response of the polycrystal. The displacement field is assumed to be of the form

$$
\underline{\boldsymbol{u}}(x)=\underset{\sim}{\boldsymbol{E}} \cdot \underline{\boldsymbol{x}}+\underline{\boldsymbol{v}}(x),
$$

with the fluctuation $\underline{v}$ periodic, meaning that it takes identical values at homologous points of the unit cell [25]. The plastic micro-deformation field, $\chi^{p}$, is assumed to be periodic, meaning that no rotational macroscopic plastic deformation is imposed to the unit cell. Its components are equal at homologous opposite nodes. According to periodic homogenization, the simple and double tractions $\underline{\boldsymbol{t}}$ and $\underset{\sim}{\boldsymbol{m}}$ are anti-periodic at homologous points of the unit cell.

Polycrystals are random materials so that the periodicity constraint may lead to a bias in the estimation of the effective properties. This boundary effect can be alleviated by considering several realization of the microstructure and performing ensemble averaging [4]. 


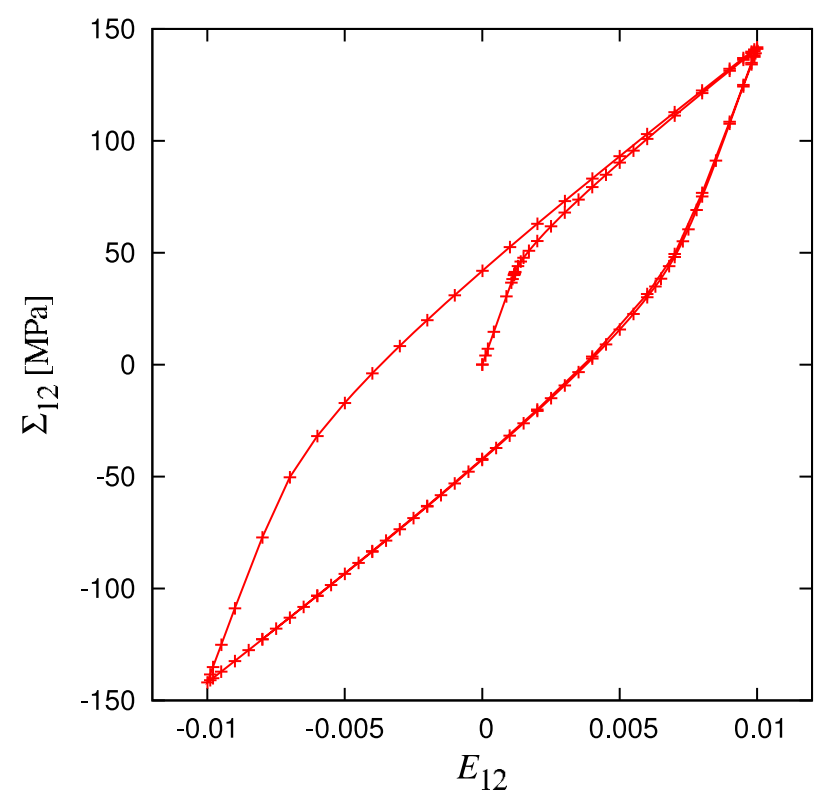

Fig. 2 (online colour at: www.gamm-mitteilungen.org) Macroscopic stress-strain response of the 24grain aggregate under cyclic simple shear loading conditions with a mean gain size $d \approx 0.2 \mu \mathrm{m}$. The set of material parameters used is labelled (c) in Table 1.

Regarding the finite element implementation of the model, 2 displacement and four plastic microdeformation degrees of freedom are attributed to each node of the mesh. Usual quadratic shape functions and their derivatives are used to evaluate the microdeformation, the strain tensor and the microdeformation gradient at the Gauss points where the evolution equations for internal variables are solved using a fourth order Runge-Kutta method. An estimate of the consistent tangent matrix is provided for the resolution of the Newton algorithm associated with the static equilibrium condition corresponding to an implicit scheme $[6,26]$. Note that this finite element formulation is probably not well-suited to the corresponding mathematical problem since only the curl of the microdeformation is considered instead of its full gradient. As a result the proposed finite element formulation may preclude localization modes which do not give rise to curl effects. For example, shear bands with strain discontinuities may not be well captured by the proposed elements which assumes continuity of the degrees of freedom. As a check of the numerical scheme, exact analytical solutions have been tested for single and double symmetric slip, see [1]. Also, we will show at the end of the paper that strain localization effects are indeed observed using the numerical scheme, see also [27]. Probably, the best numerical scheme for such a model based on the curl operator is provided in [28].

\subsection{Overall stress and cyclic response of a polycrystalline aggregate}

The finite element simulations of the boundary value problem presented previously have been conducted under generalised plane strain conditions on aggregates with a relatively small number of grains. The aim here is not to obtain a representative response but to catch the 
Table 1 Sets of material parameters used in the 24-grain aggregate case (Fig 1(a)). The intrinsic length scale, $l_{s}=\sqrt{A / H_{\chi}}$, is given for each set.

\begin{tabular}{|c|cc|cc|c|}
\hline Set & shear modulus $\mu[\mathrm{MPa}]$ & $\tau_{c}[\mathrm{MPa}]$ & $H_{\chi}[\mathrm{MPa}]$ & $A\left[\mathrm{MPa} \mathrm{mm}^{2}\right]$ & $l_{s}[\mu \mathrm{m}]$ \\
\hline $\mathrm{a}$ & 35000 & 40 & $3.010^{6}$ & $1.010^{-2}$ & $5.810^{-2}$ \\
$\mathrm{~b}$ & 35000 & 40 & $1.010^{6}$ & $1.010^{-2}$ & $1.010^{-1}$ \\
$\mathrm{c}$ & 35000 & 40 & $3.510^{5}$ & $1.010^{-2}$ & $1.710^{-1}$ \\
$\mathrm{~d}$ & 35000 & 40 & $8.810^{4}$ & $1.010^{-3}$ & $1.110^{-1}$ \\
\hline
\end{tabular}

grain size effects and to explore qualitatively the impact of different sets of material parameters. In this section, a virtual material is considered with various intrinsic length scales. The macroscopic stress-strain curve shown in Fig. 2 is obtained by applying a cyclic simple shear loading controlled by the average stress component $E_{12}$ on the aggregate of 24 grains with $d=0.2 \mu \mathrm{m}$ and the set of material parameters labelled (c) in Table 1 . The mean stress component $\Sigma_{12}$ is then computed:

$$
\Sigma_{12}=\frac{1}{V} \int_{V} \sigma_{12} d V, \quad E_{12}=\frac{1}{V} \int_{V} \varepsilon_{12} d V
$$

where $V$ denotes each polycrystal unit cell. The simulated response illustrates the kinematic hardening produced by the microcurl model. The stress-strain curves obtained in the next case (see Fig. 4) show that this kinematic hardening is size dependent: It increases for smaller grains. Note that the observed overall kinematic hardening has two distinct sources: the intragranular back-stress induced by plastic strain gradients, and the intergranular internal stress that originates from the grain to grain plastic strain incompatibilities. The latter contribution is also predicted by classical crystal plasticity models.

Figure 3 presents the effect of the mean grain size, $d$, on the macroscopic flow stress at $1 \%$ plastic strain in the 24-grain aggregate in a log-log diagram for different intrinsic length scales, $l_{s}$, introduced through the sets of material parameters (labelled a, b, c and d) given in Table 1. The curves exhibit two plateaus for large $(d>20 \mu \mathrm{m})$ and small $(d<0.1 \mu \mathrm{m})$ mean grain sizes with a transition domain in between. This tanh-shape indicates that when $d$ is large compared to the intrinsic length scale, $l_{s}$, strain gradient effects are small and the kinematic hardening arising from the microcurl model vanishes. The model saturates when $d$ is of the order of $l_{s}$ or smaller. The transition domain exhibits a strong size dependence, the polycrystalline aggregate becoming harder for decreasing grain sizes. The position of the transition zone, the maximum extra-stress (the distance between the two plateaus) and the scaling law exponent, $m$, in the size dependent domain are controlled by the material parameters used in the model. The two latter effects are controlled by the coupling modulus, $H_{\chi}$, they both increase for higher values of $H_{\chi}$ as shown in Fig. 3. The scaling exponent is defined as the slope in the log-log diagram in the inflection domain, reflecting the scaling law:

$$
\Sigma_{12} \propto d^{m}
$$

It is obtained with the sets of material parameters given in Table 1. The found values range from -0.26 to -0.64 including the well-known Hall-Petch exponent $m=-0.5$. In fact it 


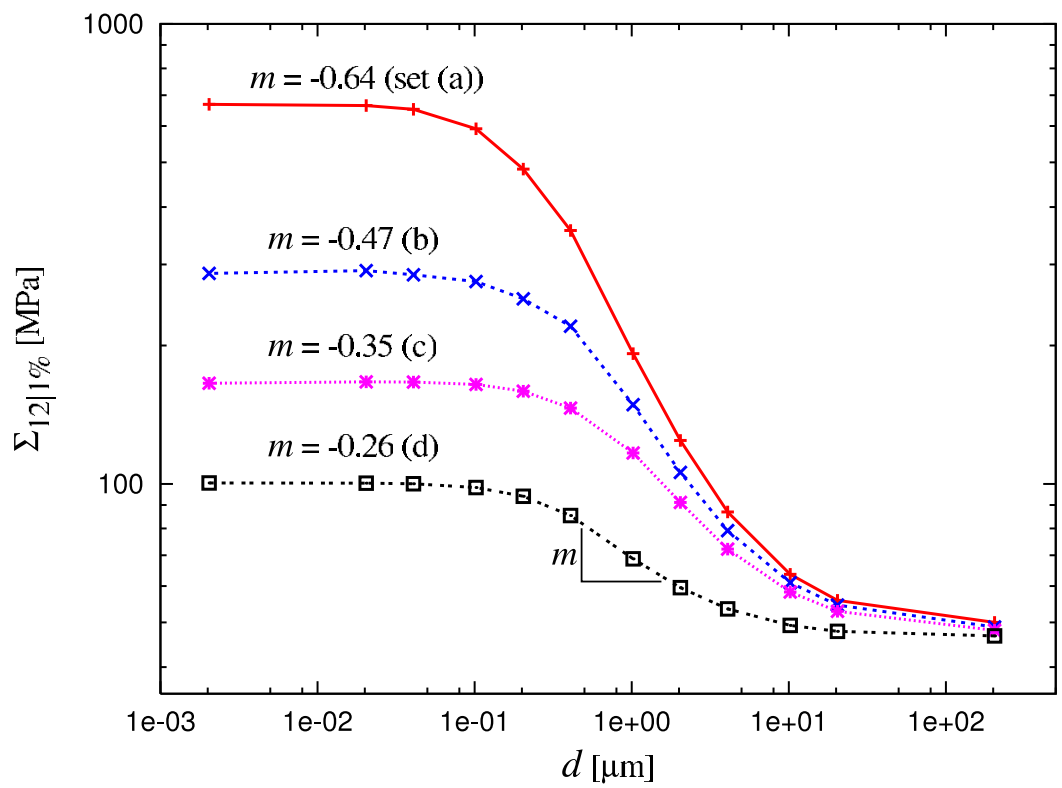

Fig. 3 (online colour at: www.gamm-mitteilungen.org) Effect of the mean grain size, $d$, on the macroscopic flow stress, $\Sigma_{12 \mid 1 \%}$, at $1 \%$ plastic strain. The results are obtained for the 24 -grain aggregate using the sets (a) to (d) of material parameters given in Table 1. The scaling law exponent, $m$, is identified in each case.

was shown in [1] that values of $m$ ranging from 0 to -2 can be simulated with the microcurl model in the case of two-phase microstructures. In each case, these values are obtained without classical isotropic hardening, meaning that the linear kinematic hardening produced by the model is able to reproduce a wide range of scaling laws. Note that conventional strain gradient plasticity models do not lead to tanh-shape curves but rather to unbounded stress increase for vanishingly small microstructures [1].

\section{Grain size effects in idealised aluminium polycrystals}

Similar finite element simulations have been performed on idealised aluminium aggregates of 52 grains shown in Fig. 1(b). An additional isotropic hardening component is added as in [29] to obtain a more realistic response of large aluminium grains. The size-independent hardening law reads:

$$
R^{\alpha}=\tau_{c}+Q \sum_{\beta}^{n} h^{\alpha \beta}\left(1-\exp \left(-b \gamma_{\text {cum }}^{\beta}\right)\right),
$$

where $n$ is the number of slip systems (here $n=2$ ), $Q$ and $b$ are material coefficients defining non-linear isotropic hardening, $h^{\alpha \beta}$ is the interaction matrix and $\gamma_{\text {cum }}^{\beta}$ is the accumulated micro-plastic slip on the slip system $\beta$. Cumulative plastic slip results from the integration of the differential equation $\dot{\gamma}_{\text {cum }}^{\beta}=\left|\dot{\gamma}^{\beta}\right|$. The material parameters used in these simulations 


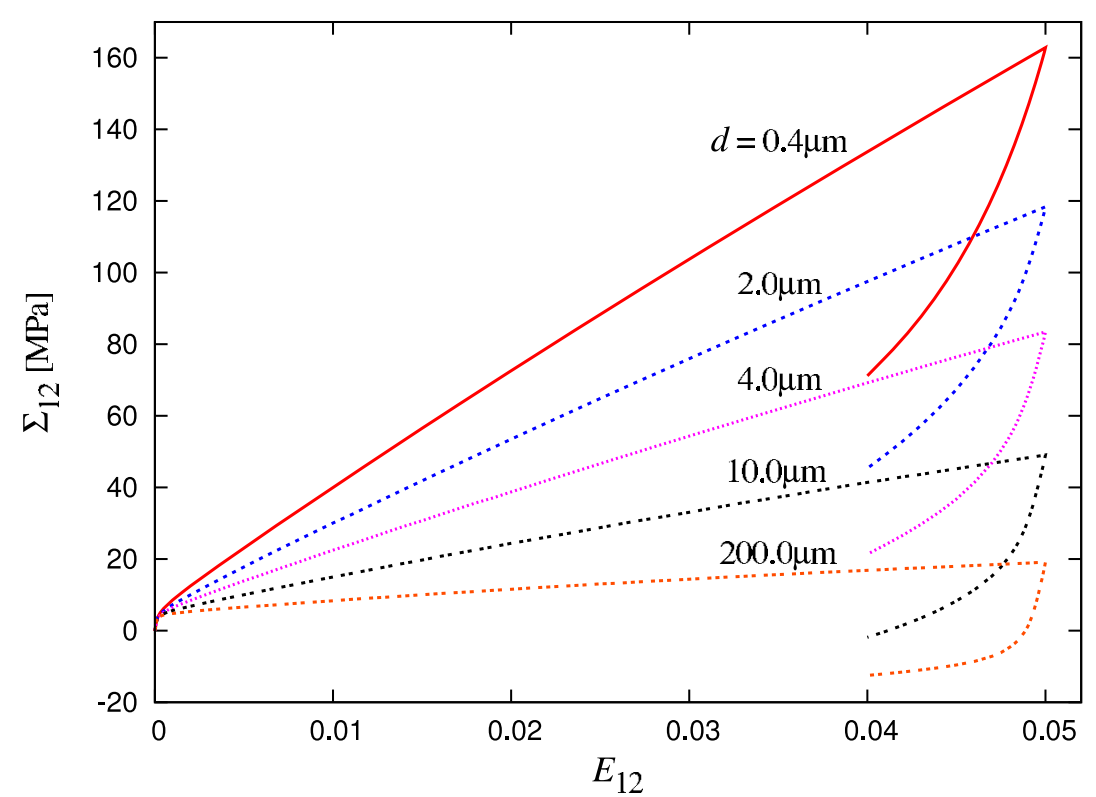

Fig. 4 (online colour at: www.gamm-mitteilungen.org) Macroscopic stress-strain response of the 52grain aggregate under simple shear for various mean grain sizes, $d$. The set of material parameters used is labelled $(\mathrm{g})$ in Table 2.

Table 2 Sets of material parameters used in the 52-grain aggregate case (Fig 1(b)).

\begin{tabular}{|c|cccccc|cc|c|}
\hline Set & $\mu[\mathrm{MPa}]$ & $\tau_{c}[\mathrm{MPa}]$ & $Q[\mathrm{MPa}]$ & $b$ & $h^{\alpha \alpha}$ & $h^{\alpha \beta, \alpha \neq \beta}$ & $H_{\chi}[\mathrm{MPa}]$ & $A\left[\mathrm{MPa} \mathrm{mm}^{2}\right]$ & $l_{s}[\mu \mathrm{m}]$ \\
\hline $\mathrm{e}$ & 27000 & 0.75 & 7.9 & 10.2 & 1 & 4.4 & $1.010^{6}$ & $1.010^{-2}$ & $1.010^{-1}$ \\
$\mathrm{f}$ & 27000 & 0.75 & 7.9 & 10.2 & 1 & 4.4 & $3.510^{5}$ & $1.010^{-2}$ & $1.710^{-1}$ \\
$\mathrm{~g}$ & 27000 & 0.75 & 7.9 & 10.2 & 1 & 4.4 & $5.010^{4}$ & $1.010^{-2}$ & $4.510^{-1}$ \\
\hline
\end{tabular}

are given in Table 2. The macroscopic stress-strain curves presented in Fig. 4 are obtained by applying a simple shear loading controlled by the average strain component $E_{12}$ on the 52 -grain aggregate with various mean grain sizes, $d$, taken in the size dependent domain. The chosen set of material parameters has the label $(\mathrm{g})$ in Table 2. These parameters are such that an acceptable description of aluminium polycrystals is obtained for large grains and that a Hall-Petch-like behaviour is found in a plausible range of grain sizes. However we did not attempt to calibrate the amplitude of the extra-hardening so that simulation predictions remain qualitative. The curves of Fig. 4 show again that the kinematic hardening produced by the model is strongly size dependent. The evolution of the macroscopic flow stress at $1 \%$ plastic strain in the 52-grain aggregate is shown in Fig. 5 in the same way as it was done in Fig. 3. The set of material parameters ( $g$ ) of Table 2 gives the ideal Hall-Petch scaling law exponent $m=-0.5$. 


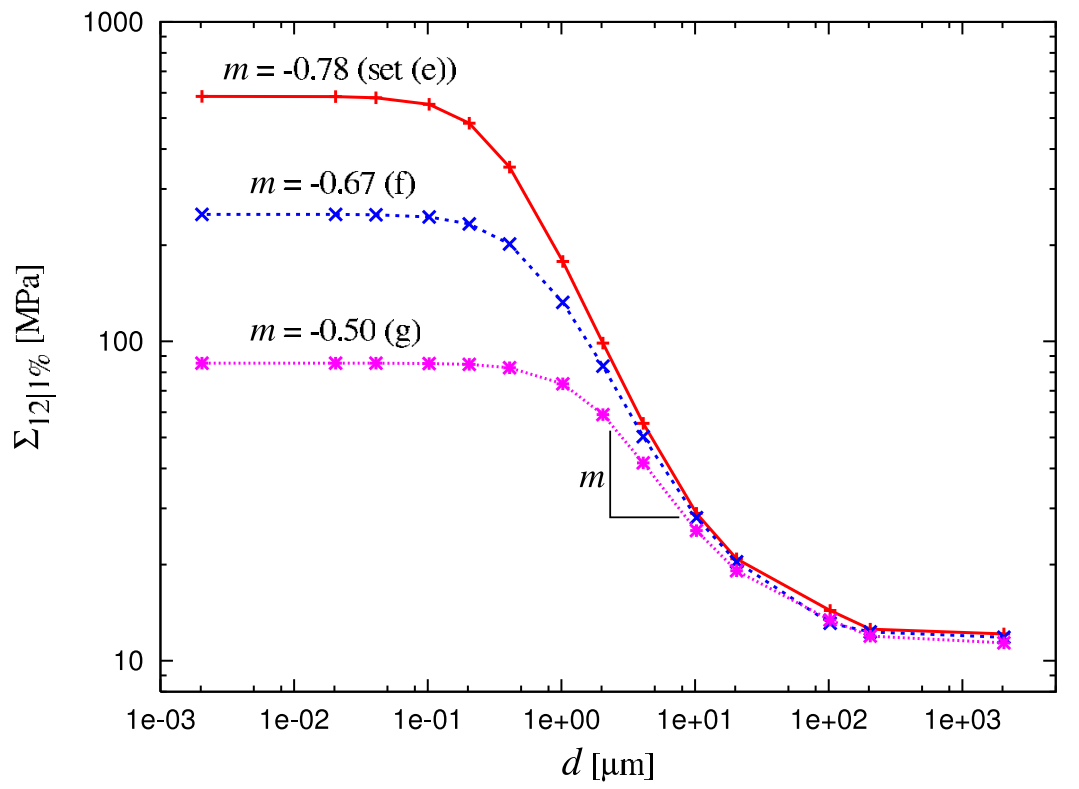

Fig. 5 (online colour at: www.gamm-mitteilungen.org) Effect of the mean grain size, $d$, on the macroscopic flow stress, $\Sigma_{12 \mid 1 \%}$, at $1 \%$ plastic strain. The results are obtained for the 52 -grain aggregate using the different sets of material parameters given in Table 2. The scaling law exponent, $m$, is identified in each case.

An important output of the simulations is the dependence of the stress and strain fields in the grains of the polycrystal on grain size. Figures 6 and 7 show the contour plots of the field of accumulated plastic slip, computed as

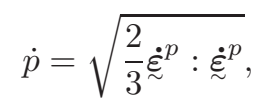

where ${\underset{\sim}{\varepsilon}}^{p}$ is the symmetric part of the plastic deformation, ${\underset{\sim}{\boldsymbol{H}}}^{p}$, and the contour plots of the norm $\Gamma$ of the dislocation density tensor,

$$
\Gamma=\sqrt{\underset{\sim}{\Gamma}: \underset{\sim}{\Gamma}}
$$

respectively. The considered grain sizes are taken in the size dependent domain where the evolution of the fields is assumed to be physically relevant. The chosen set of material parameters has the label $(\mathrm{g})$ in Table 2, it corresponds to an intrinsic length scale $l_{s}=0.45 \mu \mathrm{m}$ and gives a scaling law exponent $m=-0.5$. The mean value of the accumulated plastic slip is the same in every case, only its distribution varies with the size of the microstructure as shown in Fig. 6.

The first contour plot of each figure is obtained for $d=200 \mu \mathrm{m} \gg l_{s}=0.45 \mu \mathrm{m}$, at the very beginning of the size-dependent behaviour domain according to Fig. 5. At this size, the simulated fields show that $p$ is quite inhomogeneous and that some deformation bands appear; $\Gamma$ is localised at the grain boundaries and almost vanishes in the grain cores. The 

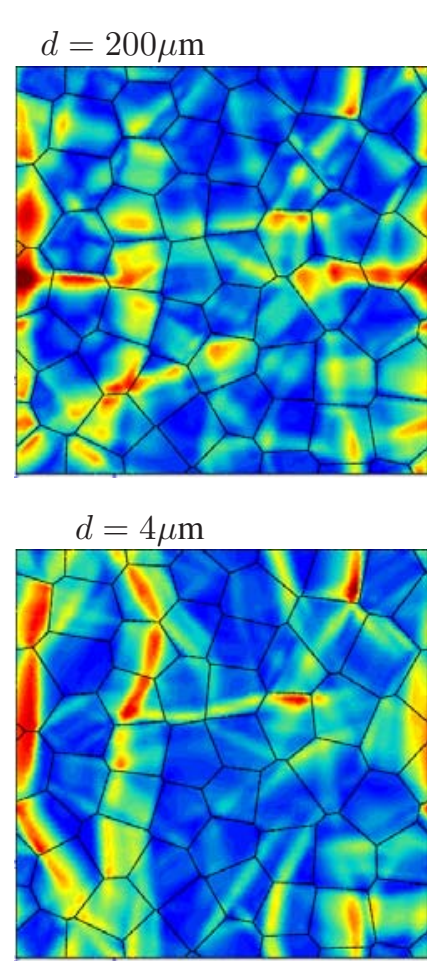
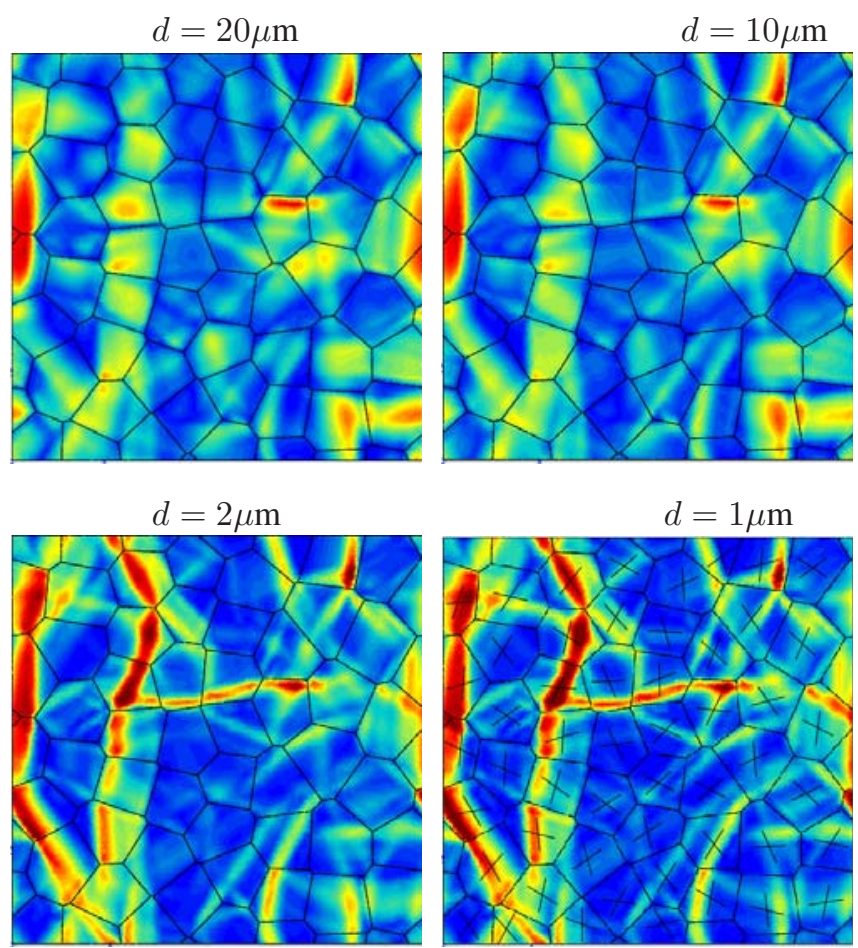

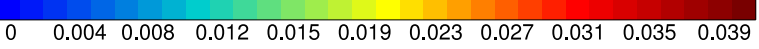

Fig. 6 (online colour at: www.gamm-mitteilungen.org) Grain size effect on the accumulated plastic slip. These contour plots are obtained with the 52-grain aggregate for the same mean value of $p=0.01$. The set of material parameters $(\mathrm{g})$ of Table 2 is used. The pairs of slip plane directions are represented for each grain on the $1 \mu \mathrm{m}$ contour plot.

contour plots obtained for $2 \mu \mathrm{m}<d<20 \mu \mathrm{m}$ show a significant evolution of both fields. One observes the progressive building of a network of strain localization bands. These bands are slip bands as they are parallel to the slip plane directions represented on the $1 \mu \mathrm{m}$ contour plot of Fig. 6. They compensate the larger blue zones where plastic strain cannot develop due to the higher energy cost associated with its gradient. Plastic strain becomes stronger inside the localization bands. The snapshots of Fig. 6 are given for a mean value of cumulative plastic strain $p=0.01$. They correspond to different values of the imposed total shear strain since the overall elastic part of strain increases for decreasing grain sizes, as suggested by Fig. 4. The field of the norm of the dislocation density tensor is still high close to grain boundaries and spreads over the grain cores. The last contour plot of each figure is obtained for $d=1 \mu \mathrm{m}$, a size close to $l_{s}$. Here the model starts to saturate, which can be seen from the simulated fields. The field of $p$ does not evolve anymore and $\Gamma$ decreases. In fact, as $l_{s}$ controls the strain gradient effects, strong strain gradients cannot develop because they become energetically too expensive when the microstructure size is too small. 

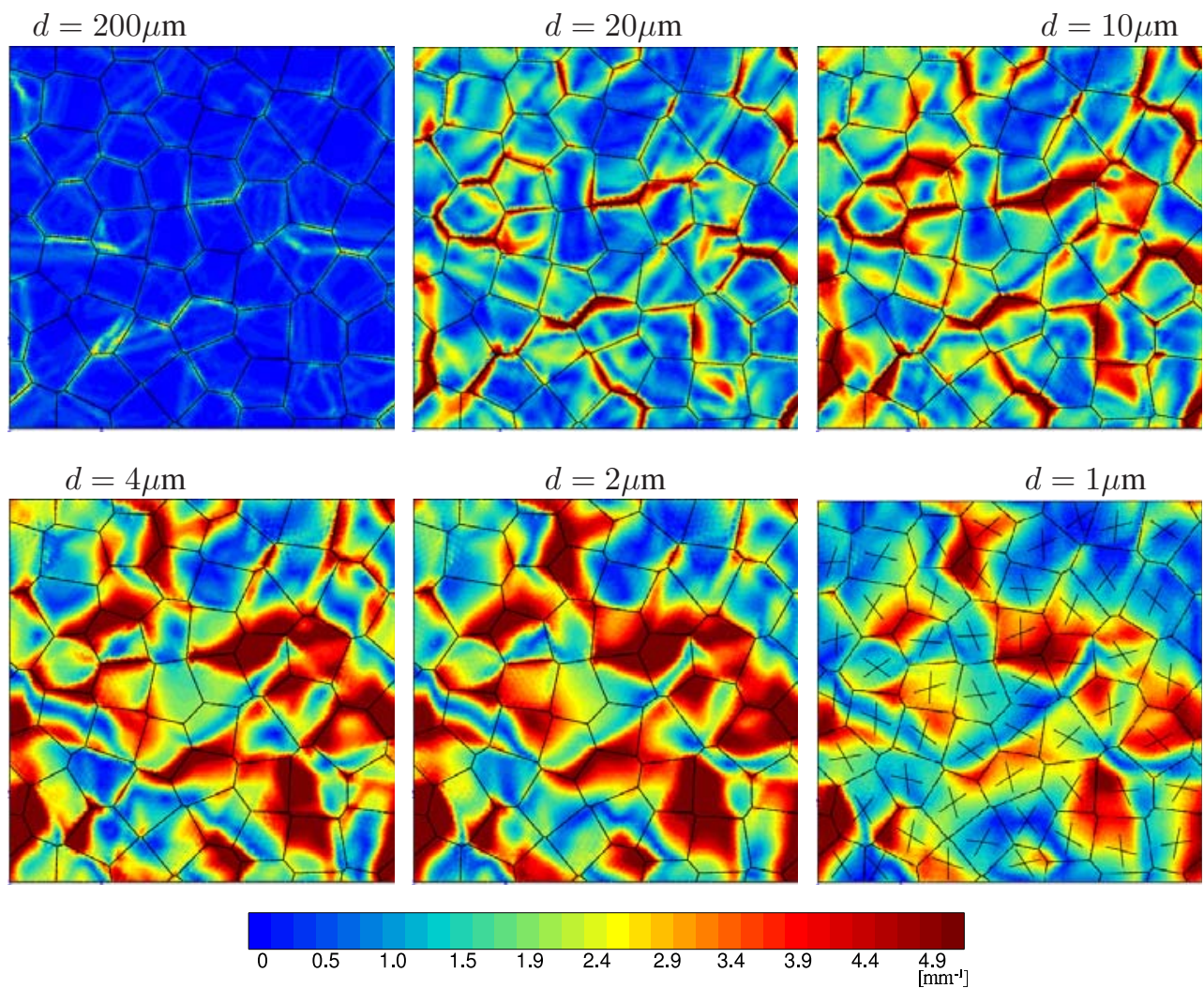

Fig. 7 (online colour at: www.gamm-mitteilungen.org) Grain size effect on the norm of the dislocation density tensor. These contour plots are obtained with the 52-grain aggregate for the same mean value of $p=0.01$. The set of material parameters $(\mathrm{g})$ of Table 2 is used. The pairs of slip plane directions are represented for each grain on the $1 \mu \mathrm{m}$ contour plot.

\section{Conclusion}

The aim of this work was to investigate the grain size effects in generalised continuum crystal plasticity. For that purpose, the microcurl model has been presented for crystal plasticity and some of its capabilities have been illustrated. It produces a linear kinematic hardening coming from the dislocation density tensor, or equivalently from the GNDs. This model was first applied to simulate small polycrystalline aggregates of 24 grains under simple shear loading. Several sets of material parameters have been used to explore their effects on the overall response. In particular, the effect of the modulus $H_{\chi}$ on the scaling law exponent was highlighted. This first set of simulations has shown that a wide range of scaling law exponents can be simulated by the model without classical isotropic hardening. Then, the simple shear response of larger idealised aluminium aggregates of 52 grains has been simulated with material parameters chosen according to the previous results. It was shown that the grain size has a strong effect on the overall behaviour of these polycrystals. Material parameters were 
set to retrieve the Hall-Petch scaling law. Note however that the simulated size effects mainly affect the hardening behaviour of the considered polycrystals and not the initial yield stress, which is a limitation of the presented constitutive framework, see the discussion in [30]. The parameter $A$ that incorporates the intrinsic length of the model, can be calibrated from the macroscopic dependence of the stress as a function of grain size for instance. So it can be calibrated from an experimental Hall-Petch diagram. Its order of magnitude can also be taken from idealized dislocation models like pile-up structures as done in [21]. The parameter $H_{\chi}$ can be used either as a penalization term to reproduce a strain gradient model but we think that it is in fact a constitutive modulus that can be identified to better reproduce the scaling law of Hall-Petch diagrams or other scaling effects, as done in [1].

A strong dependence on the size is also observed regarding plastic strain and dislocation density tensor fields. A network of slip bands is progressively built and the GND pileups, defined as the norm of the dislocation density tensor simulated with the microcurl model, spread over the grains for smaller sizes of the microstructure.

The micromorphic approach has strong computational advantages compared to strict strain gradient models for which the yield condition becomes a partial differential equation. Handling an independent set of degrees of freedom and enforcing a constraint only in a weak form makes the finite element formulation straightforward as recognized in [17, 19, 31,32].

There are still some severe limitations in the proposed approach in the sense that the constitutive equations are still naive, especially the choice of the quadratic potential with respect to the dislocation density tensor only accounts for linear kinematic hardening, as demonstrated in [1]. Non-linear evolution equations are needed for identification from experimental stressstrain loops for polycrystals. It was already mentioned in [21,33] that the modulus $A$ also depends on the dislocation density itself, as shown in [34,35].

The simulations presented in this work were limited to double slip. Considering the 12 slip systems of FCC crystals leads to significantly weaker size effects, as evidenced in [30]. In that case, the interface continuity conditions may well be insufficient to trigger the formation of boundary layers at grain boundaries.

Another perspective is the choice of an additional dependence of the free energy function on the norm of the dislocation density tensor, as used in [36,37]. In the latter reference, a direct effect of the dislocation tensor on the microscopic yield stress inside a grain was demonstrated, instead of the influence on the hardening modulus exhibited by the present model. Probably both contributions must be combined and the corresponding scaling laws remain to be determined.

\section{Acknowledgement}

Acknowledgements This work is part of the NANOCRYSTAL project ANR-07-BLAN-0186. Financial support of ANR is gratefully acknowledged.

\section{References}

[1] N. Cordero, A. Gaubert, S. Forest, E. Busso, F. Gallerneau, and S. Kruch, Journal of the Mechanics and Physics of Solids 58, 1963-1994 (2010).

[2] D. Mika and P. Dawson, Materials Science and Engineering A257, $62-76$ (1998). 
[3] F. Barbe, L. Decker, D. Jeulin, and G. Cailletaud, International Journal of Plasticity 17, 513-536 (2001).

[4] A. Zeghadi, S. Forest, A. F. Gourgues, and O. Bouaziz, Philosophical Magazine 87, 1425-1446 (2007).

[5] L. St-Pierre, E. Héripré, M. Dexet, J. Crépin, G. Bertolino, and N. Bilger, International Journal of Plasticity 24, 1516-1532 (2008).

[6] S. Forest, F. Barbe, and G. Cailletaud, International Journal of Solids and Structures 37, 71057126 (2000).

[7] K. Cheong, E. Busso, and A. Arsenlis, International Journal of Plasticity 21, 1797-1814 (2005)

[8] C. Bayley, W. Brekelmans, and M. Geers, Philosophical Magazine 87, 1361-1378 (2007).

[9] N. M. Cordero, S. Forest, E. P. Busso, S. Berbenni, and M. Cherkaoui, Grain size effect in generalized continuum crystal plasticity, in: Plasticity of crystalline materials, edited by I. R. Ionescu, S. Bouvier, O. Cazacu, and P. Franciosi (ISTE-Wiley, 2011), pp. 101-122.

[10] N. M. Cordero, S. Forest, E. P. Busso, S. Berbenni, and M. Cherkaoui, Computational Materials Science 52, 7-13 (2012).

[11] A. Eringen, Microcontinuum field theories (Springer, New York, 1999).

[12] S. Forest, Journal of Engineering Mechanics 135, 117-131 (2009).

[13] M. Gurtin, Journal of the Mechanics and Physics of Solids 50, 5-32 (2002).

[14] O. Aslan, N. M. Cordero, A. Gaubert, and S. Forest, International Journal of Engineering Science 49, 1311-1325 (2011).

[15] J. Nye, Acta Metall. 1, 153-162 (1953).

[16] P. Cermelli and M. Gurtin, Journal of the Mechanics and Physics of Solids 49, 1539-1568 (2001).

[17] S. Forest, ASCE Journal of Engineering Mechanics 135, 117-131 (2009).

[18] M. Gurtin and L. Anand, Journal of the Mechanics and Physics of Solids 57, 405-421 (2009).

[19] L. Anand, O. Aslan, and S. Chester, International Journal of Plasticity 30-31, 116-143 (2012).

[20] M. Gurtin and L. Anand, Journal of the Mechanics and Physics of Solids 56, 184-199 (2008).

[21] S. Forest and R. Sedláček, Philosophical Magazine A 83, 245-276 (2003).

[22] S. Bargmann, M. Ekh, K. Runesson, and B. Svendsen, Philosophical Magazine 90, 1263-1288 (2010).

[23] R. Asaro, Journal of applied mechanics 50, 921 (1983).

[24] V. Bennett and D. McDowell, Engineering Fracture Mechanics 70(2), 185-207 (2003).

[25] S. Forest, F. Pradel, and K. Sab, International Journal of Solids and Structures 38, 4585-4608 (2001).

[26] J. Besson, G. Cailletaud, J. L. Chaboche, S. Forest, and M. Blétry, Non-Linear Mechanics of Materials (Series: Solid Mechanics and Its Applications, Vol. 167, Springer, ISBN: 978-90-4813355-0, 433 p., 2009).

[27] H. J. Chang, A. Gaubert, M. Fivel, S. Berbenni, O. Bouaziz, and S. Forest, Computational Materials Science 52, 33-39 (2012).

[28] P. Neff, A. Sydow, and C. Wieners, Int. J. for Numerical Methods in Engineering 77, 414-436 (2009).

[29] L. Méric, P. Poubanne, and G. Cailletaud, Journal of Engineering Materials and Technology 113, 162 (1991).

[30] N. M. Cordero, S. Forest, and E. P. Busso, Comptes Rendus Mécanique 340, 261-274 (2012).

[31] R. Engelen, M. Geers, and F. Baaijens, International Journal of Plasticity 19, 403-433 (2003).

[32] S. Wulfinghoff and T. Boehlke, Proceedings of the Royal Society A 468, 2682-2703 (2012).

[33] S. Forest, Philosophical Magazine 88, 3549-3563 (2008).

[34] I. Groma, F. Csikor, and M. Zaiser, Acta Materialia 51, 1271-1281 (2003).

[35] L. Scardia, R. Peerlings, M. Peletier, and M. Geers, submited (2012).

[36] S. Conti and M. Ortiz, Arch. Rational Mech. Anal. 176, 103-147 (2005).

[37] D. Okumura, Y. Higashi, K. Sumida, and N. Ohno, International Journal of Plasticity 23, 11481166 (2007). 